\title{
The angular disclination
}

\section{MARIA COMNINOU}

Department of Applied Mechanics and Engineering Science, The University of Michigan, Ann Arbor, Michigan 48109, USA

(Received January 19, 1976)

\begin{abstract}
Similarly to the angular dislocation introduced by Yoffe, the angular disclination is a basic configuration that is suitable for generating polygonal loops by superposition. The displacements in an unbounded elastic material are given and the generation of closed loops discussed.
\end{abstract}

\section{RÉSUMÉ}

La disclinaison angulaire est une configuration fondamentale la plus facile à construire des disclinaisons en polygone, tout comme dans le cas de la dislocation angulaire introduite par Yoffe. Nous donnons ici les déplacements dans un milieu élastique infini et discutons la méthode de construction des disclinaisons en polygone.

\section{Introduction}

Suppose that an elastic body is cut along a surface $\Sigma$, the two sides of the cut displaced with respect to each other and the material rejoined by welding, gluing or similar means. The body is then left in a state of residual stress and is said to contain a Somigliana dislocation. If the relative displacement of the two sides of the cut is of the same form in position coordinates as the infinitesimal motion of a rigid body, the operation is called a Volterra dislocation. A special property of the Volterra dislocation is that it leads to a state where all components of stress are continuous at the cut $\Sigma$. Especially in solid state physics, a Volterra dislocation that involves only a translational displacement discontinuity is called simply a dislocation, but one containing also rotation a disclination [1]. The separation of Volterra dislocations into simple dislocations and disclinations is not unique because the same infinitesimal rigid body displacement can be achieved by using different axes of rotation and adjusting its translational part. A unique decomposition of a Volterra dislocation 
into a simple dislocation and a pure disclination has been proposed by Dundurs [2] and discussed further by Kröner and Anthony [3].

The idea of an angular dislocation was first advanced by Elisabeth Yoffe [4]. The importance of this configuration lies in the fact that angular dislocations can be superposed to yield a polygonal dislocation loop of arbitrary shape. The purpose of this paper is to present the displacement fields of angular disclinations in the unbounded elastic solid. Combining these results with the expressions given by Yoffe [4], it is possible to construct the elastic fields of closed polygonal disclination loops.

\section{Approach}

The angular disclination is associated with the two coordinate systems $y_{1}, y_{2}, y_{3}$ and $\eta_{1}, \eta_{2}, \eta_{3}$, as shown in Figure 1. Thus,

$$
\begin{aligned}
& \eta=y_{1}, \\
& \eta_{2}=y_{2} \cos \gamma-y_{3} \sin \gamma, \\
& \eta_{3}=y_{2} \sin \gamma+y_{3} \cos \gamma .
\end{aligned}
$$

The displacement discontinuity is imposed on the plane region $\Sigma$ which is shaded in Figure 1. The axis of rotation for the disclination is taken at the vertex of this sectorial region, so that the displacement discontinuity in the coordinate system $y_{1}$, $y_{2}, y_{3}$ is

$$
\left[u_{i}\right]=\varepsilon_{i j k} \omega_{j} y_{k},
$$

where $\omega_{j}$ is the infinitesimal rotation of one side of the cut of $\Sigma$ with respect to the other side.

The displacement field for the angular disclination in the infinitely extended

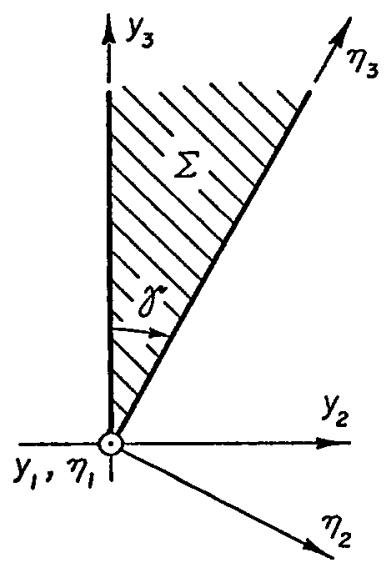

Figure 1. Angular disclination. 
material can be generated by means of the Volterra formula [5]:

$$
u_{m}(y)=\frac{1}{4 \pi} \int_{\substack{i, j, k, l, m=1,2,3\\}} \varepsilon_{i j k} \omega_{j} x_{k} \tau_{i l}^{(m)} n_{l} d S(x),
$$

where

$$
\begin{aligned}
\tau_{i l}^{m}= & (1-\alpha) r^{-3}\left[\delta_{i l}\left(x_{m}-y_{m}\right)-\delta_{l m}\left(x_{i}-y_{i}\right)-\delta_{i m}\left(x_{l}-y_{l}\right)\right] \\
& -3 \alpha r^{-5}\left(x_{i}-y_{i}\right)\left(x_{m}-y_{m}\right)\left(x_{l}-y_{l}\right), \\
r^{2}= & \left(x_{i}-y_{i}\right)\left(x_{i}-y_{i}\right), \\
\alpha= & \frac{1}{2(1-\nu)}
\end{aligned}
$$

with $\nu$ denoting Poisson's ratio, and the sign convention is the same as used by Steketee [5].

The integrals that evolve from (3) can be reduced to elementary functions. The details involving some changes of variables are straightforward, if tedious, and all intermediate steps are suppressed in this article. It might be mentioned, however, that apart from rigid body displacements, which are of no consequence, the integrals yield also displacements that correspond to homogeneous states of stress. Terms of such nature have been discarded because the principal interest in the angular disclination arises from its suitability in generating closed polygonal loops by superposition. For a closed loop, the stresses must vanish at large distances from the loop and, consequently, a final adjustment to enforce the proper boundary conditions at infinity may have to be made in each case individually.

The following notation is employed in the subsequent expressions:

$$
\begin{aligned}
& \left.\left\{Q\left(\eta_{2}, \eta_{3}\right)\right\}\right|_{0} ^{\gamma}=Q\left(\eta_{2}, \eta_{3}\right)-Q\left(y_{2}, y_{3}\right), \\
& R=\left(y_{1}^{2}+y_{2}^{2}+y_{3}^{2}\right)^{1 / 2}=\left(\eta_{1}^{2}+\eta_{2}^{2}+\eta_{3}^{2}\right)^{1 / 2}
\end{aligned}
$$

Furthermore, the multivalued function

$$
\phi=\tan ^{-1} \frac{y_{2}}{y_{1}}-\tan ^{-1} \frac{\eta_{2}}{y_{1}}+\tan ^{-1} \frac{y_{1} R \sin \gamma}{y_{1}^{2} \cos \gamma+y_{2} \eta_{2}}
$$

appears in many components of the displacements.

\section{Rotation vector $\left(\omega_{1}, 0,0\right)$}

The displacement components for this case are

$$
u_{1}=-\left.\frac{\omega_{1}}{8 \pi(1-\nu)}\left\{(1-2 \nu) \eta_{3} \ln \left(R-\eta_{3}\right)-\frac{y_{1}^{2}}{R-\eta_{3}}\right\}\right|_{0} ^{\nu},
$$




$$
\begin{aligned}
& u_{2}=\frac{\omega_{1}}{4 \pi} y_{3} \phi-\left.\frac{\omega_{1}}{8 \pi(1-\nu)} y_{1}\left\{(1-2 \nu) \sin \gamma \ln \left(R-\eta_{3}\right)-\frac{\eta_{2} \cos \gamma}{R-\eta_{3}}\right\}\right|_{0} ^{\gamma} \\
& u_{3}=-\frac{\omega_{1}}{4 \pi} y_{2} \phi-\left.\frac{\omega_{1}}{8 \pi(1-\nu)} y_{1}\left\{(1-2 \nu) \cos \gamma \ln \left(R-\eta_{3}\right)+\frac{\eta_{2} \sin \gamma}{R-\eta_{3}}\right\}\right|_{0} ^{\gamma}
\end{aligned}
$$

For the special case of $\gamma=\pi$ or a straight twist disclination,

$$
\begin{aligned}
& u_{1}=\frac{\omega_{1}}{4 \pi(1-\nu)} y_{3}\left[(1-2 \nu) \ln \rho-\frac{y_{1}^{2}}{\rho^{2}}\right], \\
& u_{2}=\frac{\omega_{1}}{2 \pi} y_{3} \tan ^{-1} \frac{y_{2}}{y_{1}}-\frac{\omega_{1}}{4 \pi(1-\nu)} \frac{y_{1} y_{2} y_{3}}{\rho^{2}}, \\
& u_{3}=-\frac{\omega_{1}}{2 \pi} y_{2} \tan ^{-1} \frac{y_{2}}{y_{1}}+\frac{(1-2 \nu) \omega_{1}}{4 \pi(1-\nu)} y_{1} \ln \rho,
\end{aligned}
$$

where

$$
\rho^{2}=y_{1}^{2}+y_{2}^{2}
$$

\section{Rotation vector $\left(0, \omega_{2}, 0\right)$}

In this case we have

$$
\begin{aligned}
u_{1}= & -\frac{\omega_{2}}{4 \pi} y_{3} \phi+\frac{\omega_{2}}{8 \pi(1-\nu)} y_{1}\left\{(1-2 \nu) \sin \gamma \ln \left(R-\eta_{3}\right)\right. \\
& \left.-\frac{y_{1}^{2} \sin \gamma}{R-\eta_{3}}+\frac{y_{3} \eta_{2}}{R\left(R-\eta_{3}\right)}\right\}\left.\right|_{0} ^{\gamma}, \\
u_{2}= & -\left.\frac{\omega_{2}}{8 \pi(1-\nu)}\left\{(1-2 \nu) \cos ^{2} \gamma\left[R+\eta_{3} \ln \left(R-\eta_{3}\right)\right]+\frac{y_{1}^{2} \cos ^{2} \gamma}{R-\eta_{3}}\right\}\right|_{0} ^{\gamma}, \\
u_{3}= & \frac{\omega_{2}}{4 \pi} y_{1} \phi+\frac{\omega_{2}}{8 \pi(1-\nu)}\{(1-2 \nu)[R \cos \gamma \sin \gamma \\
& \left.\left.-\left(\eta_{2}-\eta_{3} \cos \gamma \sin \gamma\right) \ln \left(R-\eta_{3}\right)\right]-\frac{y_{1}^{2} \cos \gamma \sin \gamma}{R-\eta_{3}}\right\}\left.\right|_{0} ^{\gamma}
\end{aligned}
$$

For $\gamma=\pi$,

$$
\begin{aligned}
& u_{1}=-\frac{\omega_{2}}{2 \pi} y_{3} \tan ^{-1} \frac{y_{2}}{y_{1}}-\frac{\omega_{2}}{4 \pi(1-\nu)} \frac{y_{1} y_{2} y_{3}}{\rho^{2}}, \\
& u_{2}=\frac{\omega_{2}}{4 \pi(1-\nu)} y_{3}\left[(1-2 \nu) \ln \rho+\frac{y_{1}^{2}}{\rho_{2}}\right], \\
& u_{3}=\frac{\omega_{2}}{4 \pi} y_{1} \tan ^{-1} \frac{y_{2}}{y_{1}}+\frac{(1-2 \nu) \omega_{2}}{4 \pi(1-\nu)} y_{2} \ln \rho .
\end{aligned}
$$


Rotation vector $\left(0,0, \omega_{3}\right)$

The results for this case are

$$
\begin{aligned}
u_{1}= & \frac{\omega_{3}}{4 \pi} y_{2} \phi+\left.\frac{\omega_{3}}{8 \pi(1-\nu)} y_{1}\left\{(1-2 \nu) \cos \gamma \ln \left(R-\eta_{3}\right)-\frac{\eta_{2} \sin \gamma}{R-\eta_{3}}\right\}\right|_{0} ^{\gamma}, \\
u_{2}= & -\frac{\omega_{3}}{4 \pi} y_{1} \phi+\frac{\omega_{3}}{8 \pi(1-\nu)}\{(1-2 \nu)[R \cos \gamma \sin \gamma \\
& \left.\left.+\left(\eta_{2}+\eta_{3} \cos \gamma \sin \gamma\right) \ln \left(R-\eta_{3}\right)\right]+\frac{y_{1}^{2} \cos \gamma \sin \gamma}{R-\eta_{3}}\right\}\left.\right|_{0} ^{\gamma}, \\
u_{3}= & -\left.\frac{\omega_{3} \sin ^{2} \gamma}{8 \pi(1-\nu)}\left\{(1-2 \nu)\left[R+\eta_{3} \ln \left(R-\eta_{3}\right)\right]+\frac{y_{1}^{2}}{R-\eta_{3}}\right\}\right|_{0} ^{\gamma}
\end{aligned}
$$

For the special case of $\gamma=\pi$ or a straight wedge disclination

$$
\begin{aligned}
& u_{1}=\frac{\omega_{3}}{2 \pi} y_{2} \tan ^{-1} \frac{y_{2}}{y_{1}}-\frac{(1-2 \nu) \omega_{3}}{4 \pi(1-\nu)} y_{1} \ln \rho, \\
& u_{2}=-\frac{\omega_{3}}{2 \pi} y_{1} \tan ^{-1} \frac{y_{2}}{y_{1}}-\frac{(1-2 \nu) \omega_{3}}{4 \pi(1-\nu)} y_{2} \ln \rho, \\
& u_{3}=0 .
\end{aligned}
$$

\section{Generation of closed loops}

The displacement discontinuity of a Volterra dislocation is of the form

$$
\left[u_{i}\right]=b_{i}+\varepsilon_{i j k} \omega_{j}\left(x_{k}-x_{k}^{0}\right),
$$

where $b_{i}$ is the translational displacement, $\omega_{i}$ the rotation, $x_{i}$ the position vector, and $x_{i}^{0}$ a constant vector on the cut $\Sigma$ which establishes the axis of rotation.

For simplicity consider a triangular loop, such as indicated in Figure 2. The figure also shows the three angular Volterra dislocations that upon adding yield the triangular loop: They are associated with the exterior angles of the loop, the cuts over which the displacements are discontinuous are indicated by shading, and the infinite parts of their legs cancel. Consequently, the area over which the displacement discontinuity appears to be imposed is exterior to the loop. However, the discontinuity can be shifted inside the loop by superposing a rigid body displacement of the semi-infinite solid above the loop with respect to that below the loop.

The position vectors of the vertices of the triangular loop are denoted by $x_{i}^{(1)}, x_{i}^{(2)}$, $x_{i}^{(3)}$. The displacement discontinuities of the angular Volterra dislocations shown are

$$
\begin{aligned}
& {\left[u_{i}^{(1)}\right]=b_{i}^{(1)}+\varepsilon_{i j k} \omega_{j}^{(1)}\left[x_{k}-x_{k}^{(1)}\right],} \\
& {\left[u_{i}^{(2)}\right]=b_{i}^{(2)}+\varepsilon_{i j k} \omega_{j}^{(2)}\left[x_{k}-x_{k}^{(2)}\right],} \\
& {\left[u_{i}^{(3)}\right]=b_{i}^{(3)}+\varepsilon_{i j k} \omega_{j}^{(3)}\left[x_{k}-x_{k}^{(3)}\right],}
\end{aligned}
$$




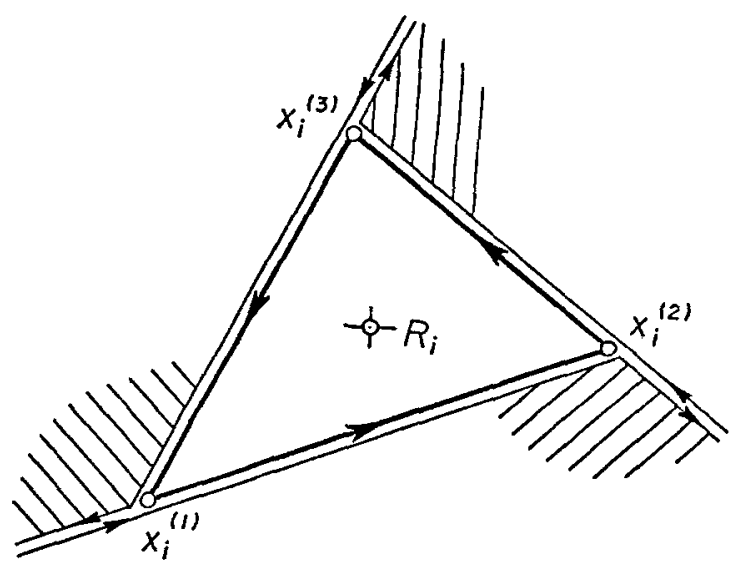

Figure 2. Triangular disclination loop.

where the superscripts refer to the three angular configurations. If these discontinuities are to add to a single rigid body displacement, the obvious requirements are that

$$
\begin{aligned}
& \omega_{j}^{(1)}=\omega_{j}^{(2)}=\omega_{j}^{(3)}=\omega_{j} \\
& b_{i}^{(1)}-\varepsilon_{i j k} \omega_{j} x_{k}^{(1)}=b_{i}^{(2)}-\varepsilon_{i j k} \omega_{j} x_{k}^{(2)}=b_{i}^{(3)}-\varepsilon_{i j k} \omega_{j} x_{k}^{(3)} .
\end{aligned}
$$

Equations (31) thus fix, say $b_{i}^{(2)}$ and $b_{i}^{(3)}$ in terms of $b_{i}^{(1)}$. However, following the Dundurs suggestion [2] of making the axis of rotation pass through the centroid of the loop fixes all translational displacements, and

$$
b_{i}^{(\alpha)}=\varepsilon_{i j k} \omega_{i}\left[x_{k}^{(\alpha)}-X_{k}\right],
$$

where $X_{k}$ is the position vector of the centroid. In addition, (32) is perfectly general and holds for a polygon with any number of sides whether plane or not.

It may also be noted that triangular Volterra dislocation loops may be used to approximate by triangulation a general Somigliana dislocation for which the displacement discontinuity is a general function of position on the cut. Therefore, the displacements given here, combined with those derived by Yoffe, can be used to construct, in a sense, a finite element scheme to treat dislocations of a general nature in the unbounded medium.

\section{REFERENCES}

[1] deWit, R., Relation between dislocations and disclinations. J. appl. Phys. 42, (1971) 3304-3307.

[2] Dundurs, J., Dislocation and disclination contents of line defects. Phys. Status Solidi (b) 53, (1972) 157-162.

[3] Kröner, E. and Anthony, K.-H., Dislocations and disclinations in material structures: The basic topological concepts. Annual review of materials science Vol. 5. Annual Reviews Inc., Palo Alto, California (1975).

[4] Yoffe, E. H., The angular dislocation. Phil. Mag. 5, (1960) 161-175.

[5] Steketee, J. A., On Volterra's dislocations in a semi-infinite elastic medium. Can. J. Phys. 36, (1958) 192-205. 